\title{
ACERCA Del Estatuto Ontológico de los Fonones
}

\author{
ABout the Ontological Status of Phonons \\ HERNAN LUCAS ACCORINTI \\ CONICET - Universidad de Buenos Aires, ARGENTINA \\ hernanaccorinti@gmail.com \\ SEBASTIAN FORTIN \\ CONICET - Universidad de Buenos Aires, ARGENTINA \\ sfortin@conicet.gov.ar
}

\begin{abstract}
Usually, a solid is conceived as a network of atoms that can vibrate around its equilibrium position generating propagating waves. However, the fact that the energy of these waves is quantized suggests an analogy with the electromagnetic field; then, the quantum "particle" called phonon is defined. In this work we study the relation between the description levels of phonons and of atoms as a case of intra-theoretical relationship.
\end{abstract}

Keywords: Intra-theoretical relationships $\bullet$ phonons $\bullet$ reductionism $\bullet$ quasiparticles $\bullet$ philosophy of chemistry

\section{Introducción}

El estudio de las relaciones interteóricas es de particular relevancia en la filosofía de la química, pues, debido al estrecho vínculo que guarda con la física, afecta la independencia y autonomía de la misma en tanto disciplina científica. La influencia que ha ejercido la física sobre la química, llegando incluso a establecerse cierta hegemonía de la primera sobre la segunda, queda manifiesta, por ejemplo, en las modernas explicaciones sobre el enlace químico surgidas a partir del advenimiento de la mecánica cuántica en la primera mitad del siglo XX. En efecto, estas explicaciones llevaron a Dirac a declarar en 1929 que la mecánica cuántica ya poseía los principios fundamentales de la química (Dirac 1929, p.714). Sin embargo, la problemática en torno a la efectiva reducción de la química a la física ha sido muy discutida recientemente por autores como Eric Scerri (2000, 2011, 2013), Robin Hendry (2010), Hinne Hettema (2012), Lombardi (2014), Lombardi y Labarca (2005) entre otros. Los principales argumentos antireduccionistas advierten que la física es incapaz de explicar la estructura de las sustancias químicas. En particular se centran en la incompatibilidad existente entre los principios fundamentales de la mecánica cuántica y el concepto de estructura molecular utilizado en química (Woolley 1978, 1982; 
Amann 1992; Sutcliffe y Wolley 2011, 2012; Bishop 2005). Sin embargo, es importante tener en consideración que las moléculas no son el único tipo de sustancia en química. Debido a ello consideramos que, para enriquecer el debate, se vuelve indispensable considerar otro tipo de sustancias como por ejemplo las sales. Las sales no son entendidas como moléculas "grandes", por el contrario, son entendidas cómo una red, que, como explicaremos en el apartado 2, es un concepto completamente diferente. En este sentido, si bien el presente trabajo no se adentrará específicamente en la discusión reduccionismo-antirreduccionismo, al brindar argumentos para una posible consolidación del estatuto ontológico de los fonones, asentará las bases para ello.

Los fonones son aquellas partículas a las que se apela para el tratamiento cuántico de las sales. Sin embargo, generalmente el fonón es concebido o bien como una pseudo-partícula, es decir un artilugio matemático necesario para realizar los cálculos, pero sin existencia "real", o bien como una cuasi-partícula, es decir una partícula no elemental que reducida en una escala temporal acotada se comporta como si fuera una de ellas (Ladyman 2015).

En este contexto el objetivo del presente trabajo será analizar el estatuto ontológico de los fonones a la luz de los argumentos usuales por medio de los que se le niega una ontología propia al fonón. Para ello, en la Sección 2, resumiremos brevemente la forma en que tradicionalmente se estudian las sales. Veremos que para dar cuenta de ciertos fenómenos, principalmente térmicos y ligados al sonido, es necesario introducir una nueva partícula: el fonón. En la Sección 3, presentaremos algunos de los éxitos empíricos que fundamentan la importancia del fonón a nivel explicativo y predictivo. En la Sección 4, analizaremos críticamente los argumentos que darían cuenta de la presunta inexistencia de los fonones. En este sentido, y a partir de una analogía establecida entre los fonones y los fotones, concluiremos que en caso de aceptarse la existencia de los fotones la misma actitud debería adoptarse para el caso de los fonones. En la Sección 5, habiendo ya demostrado que no hay suficientes razones para no considerar a los fonones como entidades existentes, exploraremos algunas posibilidades que nos permitan dilucidar su estatus ontológico. Finalmente, en la Sección 6, presentamos nuestras conclusiones.

\section{La estructura interna de los sólidos cristalinos}

Las sustancias en el estado sólido comúnmente son tratadas de manera diferencial a sus contrapartes líquidas o gaseosas, debido principalmente a la magnitud de sus componentes en el nivel molecular. Por ejemplo, un cristal macroscópico puede muy bien ser descrito como una única unidad estructural que se extiende a través de todo el cristal. Por supuesto, esta descripción repercute en las propiedades y comporta- 
miento de los sólidos a diferencia de otro tipo de compuestos. A grandes rasgos, las sustancias sólidas se pueden clasificar en dos grandes grupos, por un lado se tienen los materiales amorfos y por el otro los materiales cristalinos. Es en el segundo grupo en el se clasifican las sales y la característica distintiva de estos materiales es que sus unidades estructurales básicas posee un arreglo periódico de largo alcance.

\subsection{La descripción clásica de un sólido cristalino}

Por cuestiones expositivas haremos dos simplificaciones que no perjudicarán en modo alguno las conclusiones a las que arribaremos en el presente trabajo, ya que en caso que se desee podemos generalizar a sistemas más complejos sin mayor dificultad. Por un lado, consideraremos sólo a los llamados cristales monoatómicos, es decir, los sólidos cristalinos cuyas unidades están compuestas por un solo átomo. Por otro lado, si bien un cristal real es entendido como una red perfectamente ordenada en tres dimensiones, en este trabajo consideraremos sólo modelos en una dimensión para los sólidos cristalinos.

El modelo más simple de un sólido cristalino es una cadena infinita unidimensional de átomos unidos por medio de algún tipo de interacción. Dado que la cadena es infinita y todos sus eslabones son átomos idénticos, la cadena tiene simetría de traslación discreta. A su vez, debido al principio de razón suficiente, es de suponer que los átomos constituyentes son equidistantes en el estado de equilibrio. Ahora bien, cuando se aparta un átomo de su posición de equilibrio, entonces aparecerá una fuerza restitutiva que tienda a devolver la posición de equilibrio de dicho átomo. La fuerza restitutiva más simple es, como en un resorte, proporcional a la distancia, $F \propto d$. Si $u_{s}$ es la variable que representa la posición de la partícula $s$, y dado que esta partícula se encuentra ligada a sus vecinas $s+1$ y $s-1$, entonces la fuerza sobre ella será

$$
F_{s}=C\left(u_{s+1}-u_{s}\right)-C\left(u_{s}-u_{s-1}\right)
$$

donde $C$ es una constante de proporcionalidad. Una vez determinada la fuerza, es necesario introducirla en las ecuaciones de la mecánica clásica, por ejemplo, en la segunda ley de Newton. De este modo obtendremos una serie de ecuaciones diferenciales, si hay $N$ partículas en la cadena entonces tendremos $N$ ecuaciones como las siguientes

$$
\frac{m}{C} \frac{\partial^{2} u_{s}}{\partial t^{2}}=u_{s+1}+u_{s-1}-2 u_{s}
$$

donde $m$ es la masa de las partículas de la cadena y $s=1,2, \ldots, N$. Al resolver estas ecuaciones es posible hallar, dadas las condiciones iniciales, la expresión matemática 
que describe el movimiento de cada átomo en el tiempo. Los detalles matemáticos no son muy importantes aquí, pero sí nos interesa destacar la imagen que nos ofrece este modelo acerca de la dinámica de los sólidos: los átomos están unidos por medio de una interacción que les permite vibrar. De esta manera las vibraciones en la red cristalina forman ondas que se propagan a través del cristal por medio de las cuales son transportados el sonido y la temperatura.

\subsection{Sólidos cuánticos y el nacimiento del fonón}

El problema que nos convoca en este trabajo surge porque la descripción clásica de los sólidos cristalinos, ofrecida en el apartado anterior, no es suficiente para explicar los fenómenos que se pueden medir en el laboratorio. En particular, no puede explicar la forma que tiene la dependencia de la capacidad calorífica con la temperatura. Como veremos, para dar cuenta de ello se requerirá la introducción de una nueva entidad: los fonones.

Pero analicemos este proceso detenidamente. Considerando que la evidencia empírica sugiere que las vibraciones están cuantizadas resulta necesario cuantizar el modelo. Como las fuerzas son iguales a las del oscilador armónico, cuantizar el modelo resulta sumamente sencillo y el Hamiltoniano $\hat{H}$ se escribe como

$$
\hat{H}=\sum_{s=1}^{N} \frac{1}{2 m} \hat{p}_{s}^{2}+\frac{C}{2}\left(\hat{q}_{s+1}-\hat{q}_{s}\right)^{2}
$$

donde $\hat{p}_{s}$ y $\hat{q}_{s}$ son los operadores de momento y de posición de la partícula $s, \mathrm{y} \hat{q}_{s+1}$ es el operador de posición de la partícula $s+1$. Este es el Hamiltoniano de una cadena de partículas, en este caso átomos, que interactúan a primeros vecinos. Dado que $\mathrm{N}$ es el número total de partículas, se trata de un número realmente enorme, del orden de $10^{23}$. Por lo tanto, resolver el Hamiltoniano tal como está expresado resulta imposible. Sin embargo es posible emplear una estrategia que permite simplificar el cálculo. El primer paso consta en realizar un cambio de coordenadas especialmente elegido a las llamadas coordenadas fonónicas

$$
\hat{Q}_{k}=N^{\frac{-1}{2}} \sum_{s=1}^{N} \hat{q}_{s} e^{-i k s a} \quad \hat{P}_{k}=N^{\frac{-1}{2}} \sum_{s=1}^{N} \hat{p}_{s} e^{i k s a}
$$

donde $k=2 \pi n N^{-1} a^{-1}$ y $n=0, \pm 1, \ldots, \frac{N}{2}$. En estas coordenadas el Hamiltoniano queda escrito como una suma de osciladores armónicos que no interactúan:

$$
\hat{H}=\sum_{n} \frac{1}{2}\left(\hat{P}_{n}^{2}+\omega_{n}^{2} \hat{Q}_{n}^{2}\right)
$$


donde $\omega_{n}=(2 C / m)^{\frac{1}{2}}\left(1-\cos \left(k_{n} a\right)\right)^{\frac{1}{2}}$ es la frecuencia angular del oscilador $n$. Como el resultado obtenido muestra que el sistema se puede representar con un Hamiltoniano que exprese la suma de muchos osciladores armónicos independientes, es posible cambiar al formalismo en términos de operadores de creación y destrucción desarrollada propiamente para el oscilador armónico (Sakurai 1994). En este formalismo es posible representar a las vibraciones como partículas que se crean y se destruyen, en donde la cantidad de partículas aumentará en función del incremento de la magnitud vibracional. Así, algunos estados del sistema se pueden representar por medio de un estado $|n\rangle$, donde $n$ es un número entero que representa el número de partículas en el sistema. El operador de creación $\hat{a}^{\dagger}$, al ser aplicado a un estado del sistema con $n$ partículas devuelve otro estado con $n+1$ partículas, es decir, crea una partícula, mientras que el operador de destrucción $\hat{a}$ destruye una partícula

$$
a^{\dagger}|n\rangle=|n+1\rangle \quad a|n\rangle=|n-1\rangle
$$

Finalmente, podemos representar el Hamiltoniano en términos de estos operadores de la siguiente manera

$$
\hat{H}=\sum_{k} \hbar \omega_{k}\left(\hat{a}_{k}^{\dagger} \hat{a}_{k}+\frac{1}{2}\right)
$$

Si bien este Hamiltoniano es igual al Hamiltoniano original de la expresión (3), en esta representación ya no aparecen los átomos que introdujimos originalmente en la cadena. La nueva expresión representa a un nuevo tipo de partícula llamada fonón. Entonces dejamos de lado el modelo original en donde un cristal estaba representado por medio de una cadena de átomos unidos entre sí por medio de una interacción, para pasar a un modelo donde no hay átomos en movimiento, sino fonones. A su vez, la energía total del cristal $E$ es la suma de la energía de los fonones más una energía de "vacío" que se puede asociar a la energía de la red cuando no hay ningún fonón, es decir, cuando la red está en reposo. En efecto

$$
E=\sum_{m} \varepsilon_{m}=\sum_{m} \hbar \omega_{m}\left(n_{m}+\frac{1}{2}\right)=\sum_{m} \varepsilon^{m} n_{m}+\varepsilon_{\text {vacuum }}
$$

donde $\varepsilon^{m}$ es la energía de un fonón de frecuencia $\omega_{m}, n_{m}$ es el número de fonones de frecuencia $\omega_{m}$, y $\varepsilon_{\text {vacuum }}$ es la energía del vacío.

\section{Explicación y predicción a través de los fonones}

En el apartado anterior mostramos que mediante una serie de cálculos es posible partir del Hamiltoniano de un sistema conformado por átomos que se pueden mover interactuando a primeros vecinos y llegar al Hamiltoniano de un sistema de fonones. 
Sin embargo, lo relevante del caso y lo que hace que la pregunta por la cuestión ontológica de los fonones se imponga es que, como se adelantó en la introducción, el estudio de su comportamiento es fundamental para el estudio de las propiedades de las sales y los sólidos cristalinos en general. Es decir, los fonones desempeñan una función central en la explicación de las propiedades físicas y químicas de las sales. A modo de ejemplo presentaremos aquí dos casos simples en los que se puede apreciar el poder explicativo y predictivo del fonón.

\subsection{La ecuación del calor}

En la descripción de los sólidos cristalinos la temperatura de un sólido está dada por la presencia de fonones; cuanto mayor sea la densidad de los mismos, mayor será la temperatura. A modo ilustrativo: si tenemos una barra metálica a la que se calienta un extremo y se le enfría el otro, entonces aparece un flujo neto de calor entre los extremos de la barra que produce un gradiente de temperatura. Si aplicamos el modelo de fonones a este caso, entonces la descripción indica que en el extremo caliente hay una fuente de fonones que se trasladan a través de la barra metálica. Los fonones, al trasladarse colisionando entre sí, generan un proceso de difusión que termina con la expulsión de los mismos por el extremo frío.

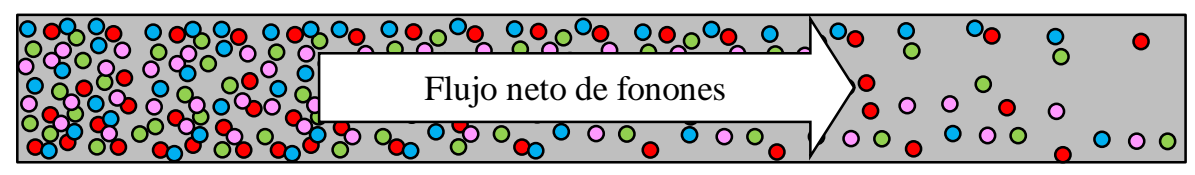

Figura 1: En una barra metálica con un extremo caliente (izquierda) donde se crean fonones y otro frío (derecha) donde se destruyen aparece un flujo neto de fonones. Como la densidad de fonones representa a la temperatura, el proceso difusivo de fonones explica el gradiente de temperatura.

De esta manera, los fonones ofrecen una imagen del flujo de calor y la temperatura basada en partículas. Dicha imagen, a nivel explicativo, es simple y muy efectiva, al menos más efectiva que la imagen abstracta del calor que ofrece la termodinámica. De hecho, resulta sorprendente que la aplicación de la mecánica cuántica a los sólidos cristalinos termine recuperando una concepción del calor similar a la que desarrollara Lavoisier en el siglo XIII con su teoría del calórico (Best 2016). En efecto, los fonones serían una versión moderna y cuantizada de la noción de la sustancia del calor.

Por otro lado, en el nivel matemático, su efectividad se hace patente al permitir deducir la bien conocida ecuación del calor. Esto se logra aplicando el modelo de fonones del siguiente modo. Si la dirección longitudinal de la barra es la dirección $x$, 
entonces, si se tienen en cuenta los choques y se aplica la teoría cinética al caso de los fonones, se obtiene que el flujo neto de energía a lo largo de la barra es

$$
j_{U}=-\frac{1}{3} C v l \frac{d T}{d x}
$$

donde $C$ es la capacidad térmica por unidad de volumen, $v$ es la velocidad media de las partículas, $l$ el recorrido libre medio de un fonón entre colisiones. (para un análisis detallado de la deducción de la ecuación del calor ver Ashcroft y Mermin 1976). Lo peculiarmente interesante de la derivación de la ecuación del calor a partir de las coordenadas fonónicas es que previamente las constantes en dicha ecuación no tenían una clara interpretación. En cambio, en términos del formalismo fonónico, la ecuación nos ofrece una adecuada interpretación de la constante al aseverar, por ejemplo, que existe una relación directamente proporcional entre la velocidad de los fonones y la propagación del calor.

\subsection{La capacidad calorífica}

Otro ejemplo interesante que refuerza la riqueza y potencialidad epistémica de los fonones es el cálculo de la capacidad calorífica de los sólidos. En este caso consideraremos que los fonones se encuentran confinados dentro de un cristal aislado sin fuentes ni sumideros de fonones. Esto permite modelar al sistema como un gas ideal de fonones confinados en una caja. Así, la energía total del cristal se puede escribir como la suma de la energía de cada uno de los fonones

$$
E=\sum_{m} \varepsilon^{m}\left\langle n_{m}\right\rangle
$$

donde $\left\langle n_{m}\right\rangle$ es el número de ocupación medio para fonones de energía $\varepsilon^{m}$ y esta dado por la distribución de Planck

$$
\left\langle n_{m}\right\rangle=\frac{1}{e^{\frac{\hbar \omega_{m}}{k_{B} T}}-1}
$$

donde $k_{B}$ es la constante de Boltzmann y $T$ es la temperatura. Entonces, es posible calcular el calor específico del cristal $C_{\text {cristal }}$ utilizando la expresión

$$
C_{\text {cristal }}=\frac{\partial U}{\partial T}
$$

Debido a la complejidad del cálculo se suelen aplicar ciertas aproximaciones. En el caso de bajas temperaturas se utiliza la aproximación de Debye y el resultado es 
proporcional al cubo de la temperatura (para los detalles de la deducción ver Kittel 1998)

$$
C_{\text {cristal }} \propto T^{3}
$$

En el caso de altas temperaturas se utiliza la aproximación de Einstein, donde la capacidad térmica resulta

$$
C_{c r i s t a l} \propto \frac{1}{T^{2}} \frac{e^{\frac{\hbar \omega}{k_{B} T}}}{\left(e^{\frac{\hbar \omega}{k_{B} T}}-1\right)^{2}}
$$

Estas ecuaciones, deducidas del formalismo fonónico, se ajustan de excelente manera con los resultados obtenidos en el laboratorio. Por ejemplo, en la Figura 2 se muestra una gráfica donde se contrastan los resultados obtenidos de la capacidad térmica del diamante experimentalmente (línea segmentada) con los resultados que es posible conseguir a través del formalismo fonónico expuesto en las ecuaciones anteriores (puntos en el gráfico). Como es posible apreciar en el gráfico, la coincidencia entre resultados experimentales y teóricos es más que aceptable. Una situación similar ocurre con el argón sólido expuesta en la Figura 3. En este caso, la coincidencia entre resultados experimentales y teóricos es excelente. Los casos anteriores, que representan sólo una muestra, nos indican que el formalismo de fonones, no es sólo un conjunto de ecuaciones estériles; si no que, por el contrario, representa una exitosa alternativa para explicar los fenómenos asociados a los sólidos demostrando éxito empírico y poder explicativo.

\section{El estatuto ontológico de los fonones}

En los apartados precedentes hemos mostrado tanto la derivación matemática de los fonones como sus virtudes explicativas. Sin embargo somos conscientes que tales virtudes epistémicas no son argumentos sólidos como para justificar la existencia de los fonones. Precisamente la tradición antirrealista apela al bien conocido argumento de la meta-inducción pesimista para afirmar que el éxito pragmático no evidencia la verdad de la teoría ni, por lo tanto, la existencia efectiva de la entidades postuladas por ella. Atentos a estas consideraciones es que en el presente apartado trataremos de demostrar, quizás no en contra de la concepción antirrealista sino de cierto tipo de realismo, que, si una adopta una actitud realista, no hay razones para no considerar a los fonones como entidades existentes. Para ello abordaremos dos estrategias. En primer lugar estableceremos una analogía con lo fotones y en segundo lugar refutaremos los criterios por los cuales suele considerarse a los fonones como entidades útiles pero irreales. 


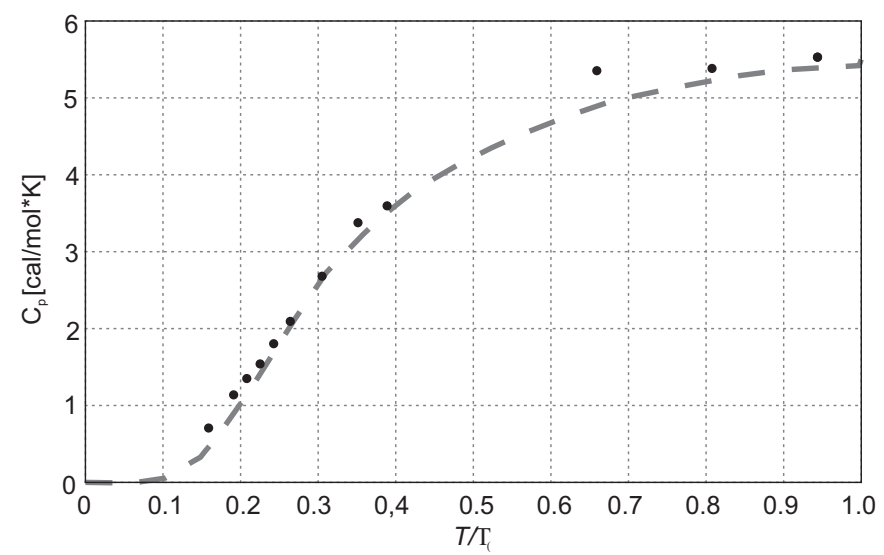

Figura 2: Comparación entre los valores experimentales de la capacidad térmica del diamante con los valores calculados con el modelo (imagen tomada de Kittel 1998).

\subsection{El fonón, un primo-hermano del fotón}

En el apartado 2.2 mostramos que mediante una serie de cálculos es posible partir del Hamiltoniano de un sistema conformado por átomos móviles y llegar al Hamiltoniano de un sistema de fonones de la siguiente manera

$\hat{H}_{\text {atoms }}+\hat{H}_{\text {int }} \stackrel{\text { Coordenadas fononicas }}{\longrightarrow} \sum_{m} \frac{1}{2}\left(\hat{P}_{m}^{2}+\omega_{m}^{2} \hat{Q}_{m}^{2}\right) \stackrel{\text { op. creación y destrucción }}{\longrightarrow} \sum_{m} \hbar \omega_{m}\left(\hat{a}_{m}^{\dagger} \hat{a}_{m}+\frac{1}{2}\right)$ donde $\hat{H}_{\text {atoms }}=\sum_{s-1}^{N} \frac{1}{2 m} \hat{p}_{s}^{2}$ y $\hat{H}_{\text {int }}=\sum_{s-1}^{N} \frac{C}{2}\left(\hat{q}_{s+1}-\hat{q}_{s}\right)^{2}$.

Lo que resulta de esta operación es sumamente relevante para el objetivo de nuestro trabajo, ya que, debido a la analogía completa, que como veremos a continuación, puede ser trazada entre los fonones y los fotones, emerge ineludiblemente la pregunta en torno al estatuto ontológico de los primeros. En efecto, los cálculos utilizados para hallar el Hamiltoniano fonónico son exactamente los mismos que se utilizan para hallar el Hamiltoniano de los fotones. Partiendo del Hamiltoniano del campo eléctrico $\hat{H}_{E}$ y $\hat{H}_{B}$, tenemos que

$$
\hat{H}_{E}+\hat{H}_{B} \stackrel{\text { Coordenadas fotónicas }}{\longrightarrow} \sum_{m} \frac{1}{2}\left(\hat{P}_{m}^{2}+\omega_{m}^{2} \hat{Q}_{m}^{2}\right) \stackrel{\text { op. creación y destrucción }}{\longrightarrow} \sum_{m} \hbar \omega_{m}\left(\hat{a}_{m}^{\dagger} \hat{a}_{m}+\frac{1}{2}\right)
$$




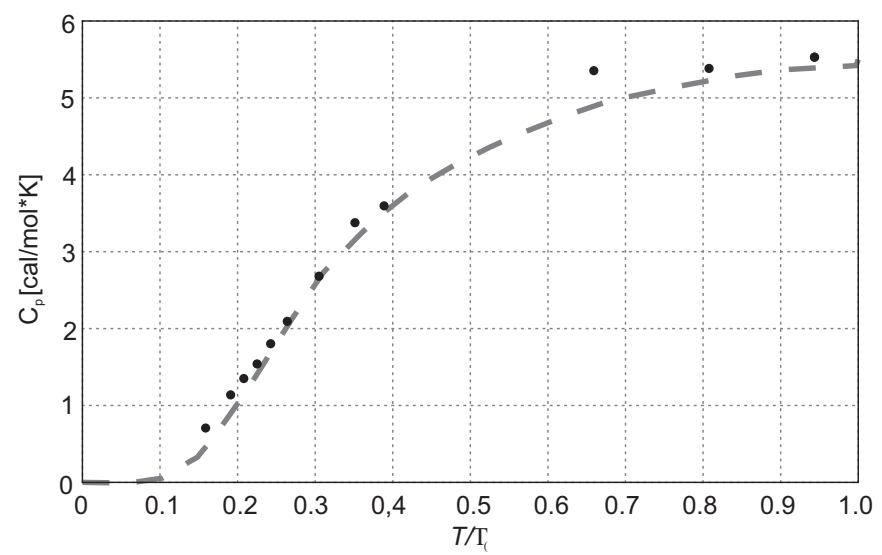

Figura 3: Comparación entre los valores experimentales de la capacidad térmica del argón a baja temperatura con los valores calculados(imagen tomada de Kittel 1998).

Es decir, siendo que la analogía entre fotones y fonones es completa, lo que nos preguntaremos es por qué habría que adscribirle al fonón el estatus ficcional o degradado de cuasi-partícula. El procedimiento de la expresión (16) nos permite entender a todas las formas de radiación electromagnética en términos de partículas; por qué el procedimiento de la expresión (15) no nos permite entender entonces a todas las formas de vibración en un cristal bajo los mismos términos?, o en otras palabras, ¿cuáles son los presupuestos por los cuales no deberíamos afirmar que la temperatura o el sonido en un cristal son manifestaciones de la presencia real de los fonones?

Es decir, en el caso de los fotones, partimos del campo electromagnético y mediante una serie de cálculos llegamos a los fotones. Posteriormente, y debido al éxito empírico, éstos adquieren luego el estatus de partículas reales. Sin embargo, para el caso de los fonones, si bien hacemos exactamente los mismos cálculos y obtenemos un éxito empírico equivalente, no se obtiene igual conclusión. En efecto, dichas partículas suelen ser consideradas como pseudopartículas o cuasipartículas (Franklin y Knox 2018; Ladyman 2015). En los apartados siguientes evaluaremos críticamente las razones para tal discriminación. En particular, trataremos de elucidar si los diferentes argumentos usualmente esgrimidos son tan poderosos como para concluir la inexistencia de los fonones.

\subsection{Dos ontologías para la materia}

A la hora de ofrecer una imagen y una matemática que describa la composición de las sustancias químicas, sin duda el modelo más popular es el que ofrece la hipóte- 
sis atómica. Es decir, el modelo según el cual la materia está compuesto por átomos móviles, en donde los fenómenos sueles explicarse a través de los átomos y su interacción. Un átomo es la unidad constituyente más pequeña de la materia que tiene las propiedades de un elemento químico y clásicamente se lo concibe como a una partícula compuesta cuyo conjunto de propiedades incluye a la posición y a la velocidad. Esta idea aplicada al caso de un sólido cristalino da lugar al modelo de la cadena de átomos que interactúan que presentamos en la Sección 2. Así, para aplicar la mecánica cuántica a una sustancia química debemos construir su Hamiltoniano como el Hamiltoniano de estos átomos más uno de interacción

$$
\hat{H}=\hat{H}_{\text {atomos }}+\hat{H}_{i n t}
$$

Con este Hamiltoniano más la aplicación de alguna aproximación que simplifique los cálculos, podemos explicar y predecir muchos fenómenos en la naturaleza. Por ejemplo, podemos calcular los niveles energéticos de una molécula (Sutcliffe y Wolley 2012).

Por otro lado, en la Sección 2 presentamos una ontología diferente para el caso de la materia en estado sólido basada en la hipótesis fonónica. De acuerdo a las ideas allí expuestas, los sólidos se componen por átomos que se encuentran en reposo y fonones que se pueden mover. A su vez, con el Hamiltoniano de estos fonones más algún Hamiltoniano de interacción es posible explicar y predecir algunos fenómenos de la naturaleza, como por ejemplo, la ecuación del calor.

A pesar de que por medio de ambas hipótesis se obtiene algún nivel de éxito empírico, en la comunidad científica prevalece la preferencia por la hipótesis atómica por sobre la fonónica. Cabe preguntarse cuáles son los motivos para esta elección.

\subsection{Dos ontologías para la materia y la supremacía de la hipótesis atómica}

A continuación identificaremos y analizaremos críticamente tres argumentos que pueden esgrimirse para sostener la hipótesis atómica usual como aquella que describe adecuadamente la realidad de los hechos en detrimento de la hipótesis fonónica.

El primer argumento, que bautizaremos como el argumento de la herramienta, plantea que para introducir los fonones se utiliza originalmente la hipótesis atómica (ver ecuación 15). Por lo tanto, los fonones serían interpretados como una mera herramienta que permitiría simplificar aquellos cálculos que no se pueden resolver directamente a partir de la hipótesis atómica.

El segundo argumento, denominado el argumento de la supercomputadora, plantea que nos vemos obligados a utilizar la hipótesis fonónica porque aún no tenemos poder de cómputo suficiente como para resolver la ecuación de Schrödinger de una 
red cristalina desde primeros principios. La presunción sería que en un futuro, cuando dispongamos de computadoras más poderosas, podremos realizar los cálculos de un modo exacto sin necesidad de apelar a tales entidades.

Finalmente, cómo último argumento, se presenta el argumento de la contidad. Lo que plantea este argumento es que la hipótesis atómica usual, a diferencia de la hipótesis fonónica, permite explicar una gran cantidad y variedad de fenómenos. En este sentido, debido a su capacidad explicativa, ambas hipótesis no estarían en el mismo nivel.

Los tres argumentos se encuentran entrelazados, ya que el argumento de la cantidad sólo tiene sentido o bien concibiendo a los fonones como meras herramientas de cálculo, o bien aferrándonos a la esperanza de que en un futuro próximo dispondremos de poder de cálculo suficiente como para prescindir de los fonones. No obstante ello, por cuestiones de claridad argumentativa, haremos un tratamiento separado de los mismos.

\subsubsection{El argumento de la herramienta}

Según el argumento de la herramienta, los fonones son introducidos, con el fin de simplificar los cálculos, partiendo del modelo de una cadena de átomos que vibran alrededor de su posición de equilibrio. En efecto, esa es la estrategia que hemos elegido en la Sección 2 y es la forma en que aparecen los fonones en los libros de texto sobre el tema (ver Ashcroft y Mermin 1976 y Kittel 1998). Sin embargo, debemos señalar que esto se hace así por cuestiones didácticas. Dado que el lector típico está familiarizado con la idea de un átomo que se puede mover, se parte de un modelo basado en ellos. No obstante, todos esos cálculos no son necesarios. Siempre es posible presentar a los fonones postulando la existencia de una nueva partícula con el Hamiltoniano

$$
\hat{H}=\sum_{k} \hbar \omega_{k}\left(\hat{a}_{m}^{\dagger} \hat{a}_{m}+\frac{1}{2}\right)
$$

En otras palabras, es posible postular la existencia de los fonones desde un principio y a partir de allí explicar las propiedades de los sólidos cristalinos. Teniendo esto en cuenta el argumento de la herramienta deja de ser un argumento en sí mismo ya que si bien los cálculos son presentados de esa manera, bien podrían presentarse de otra en la que los fonones son las entidades fundamentales. Es más, debido a que, como vimos, hay una transformación que permite pasar del Hamiltoniano de los átomos móviles al Hamiltoniano de los fonones, también hay una transformación inversa que permite pasar del Hamiltoniano de los fonones al Hamiltoniano de los átomos móviles. En efecto, es posible llegar al Hamiltoniano de los átomos móviles partiendo del Hamiltoniano de los fonones. De este modo, si se quiere podría argumentarse 
que las entidades fundamentales son los fonones y que los átomos móviles son una herramienta de cálculo útil en problemas que no involucran fenómenos térmicos. Este razonamiento muestra que el argumento de la herramienta, al ser simétrico, no se sustenta por sí sólo. Sino que podría llegar a ser tenido en cuenta a modo complementario en el caso de que se dispongan otros argumentos a favor de los átomos móviles. En caso de poder demostrar con otros argumentos que la ontología fundamental de los materiales es aquella que está basada en átomos móviles, el argumento de la herramienta explicaría por qué a veces resulta conveniente utilizar fonones.

Hasta aquí hemos mostrado que la forma de presentar a los fonones a partir de los átomos móviles no es la única posible sino que es una entre muchas que tiene la particularidad de presentar a los fonones como meras herramientas de cálculo. A continuación mostraremos que aún si concedemos este punto, el argumento sólo es válido en un caso muy particular, el caso sin interacción. La derivación de los fonones presentada en la Sección 2 parte de considerar un grupo de átomos que se relacionan entre sí por medio de una interacción cuadrática y termina en el Hamiltoniano de los fonones. Los fonones que aparecen en la derivación no interactúan entre sí, por lo tanto dicho esquema sólo es útil para describir un número limitado de situaciones más o menos idealizadas.

En el caso general, cuando queremos usar fonones para describir algún fenómeno en un cristal partimos del Hamiltoniano (18) e introducimos algún término de interacción fonónica en caso de necesitarlo. Por ejemplo, para explicar la dependencia de la resistividad eléctrica con la temperatura se considera que los electrones que se mueven en un conductor colisionan y son dispersados por los fonones de la red cristalina. Cuanto más elevada es la temperatura, más fonones existen, y por consiguiente más choques se producen. En los experimentos se mide la constante de acoplamiento electrón-fonón y a partir de ella se calcula el número de fonones que rodean a un electrón en movimiento dentro de un cristal. De este modo, es posible determinar el tipo de interacción entre las partículas involucradas (Kittel 1998). Así, para describir este fenómeno se parte del hecho de que hay fonones por un lado y electrones por otro, luego se postula que estas dos partículas pueden interactuar entre ellas y a partir de las mediciones se deduce el tipo de Hamiltoniano de interacción necesario para reproducir los experimentos. Por lo tanto el punto de partida son los fonones, no los átomos móviles.

Lo mismo ocurre con el experimento realizado por Shinen (1963) en cristales de óxido de magnesio, en donde puede observase un proceso de scattering entre fonones. En efecto, en su artículo Shinen describe un experimento en donde como consecuencia del entrecruzamiento entre dos haces de fonones con frecuencia $\omega_{1}$ y $\omega_{2}$, se produce un tercer haz con frecuencia $\omega_{1}+\omega_{2}$. Esto lo indujo a pensar que cuando un fonón con frecuencia $\omega_{1}$ choca con un fonón de frecuencia $\omega_{2}$ en un proceso de scattering donde se conserva la energía, se produce un tercer fonón 
de frecuencia $\omega_{1}+\omega_{2}$. El proceso de scatering debe darse a través de algún tipo de interacción entre los fonones; es decir, al Hamiltoniano de los fonones debe agregarse un Hamiltoniano de interacción

$$
\hat{H}=\sum_{k} \hbar \omega_{k}\left(\hat{a}_{m}^{\dagger} \hat{a}_{m}+\frac{1}{2}\right)+\hat{H}_{i n t}=\hat{H}_{\text {fotones }}+\hat{H}_{i n t}
$$

Este Hamiltoniano de interacción es el que tiene interpretación directa, como la interacción entre fonones que se mide en el experimento. A partir de las mediciones se determina cuál es el Hamiltoniano de interacción entre fonones. Luego, es posible hacer un cálculo de primeros principios en el que se vincule esta interacción con términos no cuadráticos en la red cristalina. Es decir, esa interacción entre fonones se puede reinterpretar como la introducción de un término extra, no cuadrático, en el Halimtoniano de la expresión (3). Es más, en los casos donde esto es posible aún queda pendiente brindar una explicación de por qué está ese término no cuadrático en la interacción entre los átomos. En resumen, se comienza trabajando con fonones y luego se intenta reinterpretar los resultados desde el punto de vista de los átomos móviles. Esto muestra que si bien en el caso de los fonones no interactuantes podría llegar a defenderse la postura según la cual los fonones son meras herramientas de cálculo, en el caso general dicha postura se torna insostenible. Recordemos que según el argumento de la herramienta los fonones son interpretados de un modo instrumental por el hecho de que en la presentación usual se derivan de los átomos. Sin embargo lo que aquí demostramos es que en el caso donde los fonones pueden interactuar los roles se invierten: se parte de los fonones, se trabaja con fonones, se experimenta con fonones, se determina el Hamiltoniano de interacción de los fonones y luego, recién al final, se traducen los resultados en términos de átomos móviles. En el caso con interacción el modelo de los fonones forma parte de la metodología de trabajo, se los utiliza para plantear el problema desde el principio y para diseñar los experimentos. Un ejemplo similar se puede encontrar en Kong et al. 2001, donde se calcula el acoplamiento fonón-electrón en cristales de boruro de magnesio.

Para aclarar nuestro punto, debemos mencionar que en la mayoría de las situaciones de estudio en los sólidos se debe agregar un término de interacción al Hamiltoniano. Es decir, en la práctica científica, se parte del Hamiltoniano (18) y dependiendo del sistema físico bajo estudio se agrega un término extra que de cuenta de la interacción entre fonones. Este término extra no es calculado, sino que se agrega a partir de las observaciones experimentales o bien por similitud con otros sistemas físicos. Luego, si a partir de este Hamiltoniano quisiéramos volver al formalismo estándar con átomos en movimiento para tratar de dilucidar que nos ofrece desde este formalismo el término de interacción introducido, el procedimiento se vuelve prácticamente imposible debido a la dificultad de los cálculos que se deben llevar a cabo. Además, y este es el punto importante aquí, el término de interacción tiene una 
interpretación correcta y directa dentro del formalismo fonónico; es decir, aunque logremos averiguar cuál es el término de interacción equivalente en el formalismo estándar este no nos entregaría información útil por no poseer una interpretación que aporte algún tipo de explicación. Por ejemplo, para el caso de la dependencia de la resistencia eléctrica con la temperatura el formalismo fonónico ofrece una explicación poderosa del fenómeno: al circular corriente por un sólido los electrones chochan con los fonones y cuanto mayor temperatura más fonones hay, por lo tanto cuanto mayor temperatura mayor será la resistencia del material al paso de corriente eléctrica. En cambio, desde el formalismo de la hipótesis atómica usual lo máximo que se puede decir es que esto ocurre porque hay un término no cuadrático en el Hamiltoniano.

Como afirmamos al inicio del presente apartado existen ciertas posturas de índole filosóficas y científicas que puede empañar las conclusiones que aquí intentan plantearse. Por una lado, uno puede, aun cuando se cumplan los criterios precedentemente mencionados, adoptar una actitud radicalmente antirrealista y considerar a todas las entidades como meras herramientas de cálculo. Siendo esto una actitud absolutamente plausible consideramos que la misma no atenta en modo alguno contra nuestros objetivos. En definitiva, el auditorio al que pretendemos interpelar es a aquél que, aun teniendo una actitud realista, niegan sin embargo la existencia de los fonones. En este sentido creemos que la analogía establecida en el apartado 4.1 entre los fonones y los fotones (entidades estas últimas a los que sí suele atribuirse existencia) y la demostración de que la hipótesis fonónica puede considerarse una hipótesis de primeros principios, socava la defensa de aquellos realistas que, aceptando la existencia de los fotones mantienen una actitud escéptica para con los fonones. Podríamos afirmar entonces la siguiente dicotomía: o bien admitimos un instrumentalismo general para con todas las entidades, o bien introducimos a los fonones en el campo de las entidades existentes.

Pero veamos la fuerza de los otros dos argumentos restantes. Precisamente la debilidad del argumento de la herramienta quedará de manifiesto cuando mostremos la debilidad de los otros argumentos sobre los cuales éste se sostiene.

\subsubsection{El argumento de la supercomputadora}

Este argumento insiste con que los fonones son herramientas de cálculo que podrán ser descartados cuando dispongamos de poder de cómputo suficiente para resolver de un modo exacto la ecuación de Schrödinger de los cristales desde primeros principios. Veamos esto con un poco más de detalle.

Dado un sistema cuántico $U$, de acuerdo a la mecánica cuántica el mismo tiene asociado un Hamiltoniano $\hat{H}_{U}$. Siendo así, existen muchas formas de dividir al sistema U en partes. En particular siempre es posible sumar y restar el Hamiltoniano que 
uno elija, por ejemplo $\hat{H}_{X}$ de manera que el Hamiltoniano original resulte inalterado

$$
\hat{H}_{U}=\hat{H}_{X}-\hat{H}_{X}+\hat{H}_{U}=\hat{H}_{X}+\hat{H}_{i n t}
$$

donde $\hat{H}_{\text {int }}=-\hat{H}_{X}+\hat{H}_{U}$. Este es un procedimiento sumamente artificial pero correcto, que muestra que a cualquier Hamiltoniano siempre lo podemos escribir como el Hamiltoniano que uno elija más algún término de interacción. El hecho de que esto se pueda hacer no significa, como veremos más adelante, que siempre sea útil.

De lo anterior podemos concluir que dado un sistema cuántico es posible representar su Hamiltoniano como el Hamiltoniano de átomos móviles más uno de interacción, $\hat{H}_{U}=\hat{H}_{\text {atomos }}+\hat{H}_{\text {int }}^{\prime}$. O bien representar al sistema como el Hamiltoniano de los fonones más uno de interacción distinto al del caso anterior, $\hat{H}_{U}=\hat{H}_{\text {fonones }}+\hat{H}_{\text {int }}$. Es importante notar que estos dos Hamiltonianos son exactos, ya que no hemos realizado ningún tipo de aproximación. En el caso hipotético de disponer de una supercomputadora que nos permitiera encontrar las soluciones para sistemas de muchas ecuaciones, entonces podríamos usarla, como dice el argumento que estamos considerando, para resolver el modelo construido a partir de la hipótesis atómica usual, y calcular, por ejemplo, el valor medio de algún observable $\hat{O}$. Sin embargo, nada impide que podamos utilizar la misma supercomputadora para resolver el Hamiltoniano de los fonones, calculando el valor medio del mismo observable $\hat{O}$. En ambos casos, debido a que se utilizan las soluciones exactas del mismo Hamiltoniano, obtendríamos exactamente el mismo resultado.

$$
\langle\hat{O}\rangle_{\text {atomos }}=\langle\hat{O}\rangle_{\text {fonones }}
$$

De este modo, es posible concluir que debemos descartar el argumento de la supercomputadora, porque si bien es cierto que con ella podremos describir cualquier problema con la hipótesis atómica usual, también es cierto que podremos hacer lo mismo, y con la misma efectividad, con la hipótesis fonónica. La supercomputadora es neutral en esta discusión. Es decir, considerando que estamos evaluando los argumentos en forma diferenciada, eliminar al fonón del campo de lo realmente existente no puede consustanciarse sobre la base de este argumento. Este argumento por y en sí mismo es ineficaz.

\subsubsection{El argumento de la cantidad}

Según este argumento la prioridad ontológica de los átomos móviles respecto a los fonones se debe a que los primeros explican, a diferencia de los segundos, una gran cantidad de fenómenos. 
Volvamos al ejemplo de la sección anterior en donde podemos escribir el Hamiltoniano de cualquier sistema en acuerdo con ambas hipótesis

$$
\hat{H}_{U}=\hat{H}_{\text {atomos }}+\hat{H}_{\text {int }}=\hat{H}_{\text {fonones }}+\hat{H}_{\text {int }}^{\prime}
$$

Habíamos visto que si disponemos de una supercomputadora entonces podemos calcular las soluciones exactas en ambos casos, obteniendo los mismos resultados. Pero, ¿qué pasa si no tenemos la supercomputadora?

Bueno, si no tenemos la supercomputadora entonces tendremos que emplear algún tipo de aproximación. Las aproximaciones más comunes son siempre variantes de la Teoría de Perturbaciones (Ballentine 1990). Para aplicar esta aproximación al caso del Hamiltoniano de los átomos móviles es necesario pedir que $\hat{H}_{\text {atomos }} \gg \hat{H}_{\text {int }}$ ya que, si este requisito se cumple, entonces podremos aproximar las soluciones y calcular el valor medio de algún observable . Por otro lado para poder aplicar la teoría de perturbaciones al caso del Hamiltoniano de los fonones es necesario pedir que $\hat{H}_{\text {fonones }} \gg \hat{H}_{\text {int }}^{\prime}$, ya que, nuevamente, si este requisito se cumple entonces podremos aproximar las soluciones y calcular el valor medio del mismo observable $\hat{O}$.

Sin embargo, a diferencia de lo ocurrido para el caso de la solución exacta presentada en la sección anterior, en este caso el resultado no es el mismo

$$
\langle\hat{O}\rangle_{\text {atomos }} \neq\langle\hat{O}\rangle_{\text {fonones }}
$$

Esto se debe a que se trata de soluciones aproximadas y en tanto tales siempre sucede que una solución sea mejor que la otra. Para ser más precisos, es necesario aclarar que en general no podremos aplicar la teoría de perturbaciones a los dos Hamiltonianos ya que los requerimientos $\hat{H}_{\text {atomos }} \gg \hat{H}_{\text {int }}$ y $\hat{H}_{\text {fonones }} \gg \hat{H}_{\text {int }}^{\prime}$ son incompatibles y no pueden ser satisfechos al mismo tiempo. Como consecuencia, en el caso general se podrá aplicar un modelo o el otro, pero no los dos.

Entonces, si bien es cierto que sin una supercomputadora que nos asista la hipótesis atómica usual resulta exitosa en muchos casos, hay una parte de la realidad donde el modelo que de ella se deriva no funciona. En el caso de procesos térmicos, debemos usar la hipótesis fonónica, no por capricho, sino por necesidad. No importa que la lista de zonas de la realidad donde se puede aplicar la hipótesis atómica sea más amplia que la de los fonones, ellos se complementan ya que para explicar la gama de todos los fenómenos ambas son imprescindibles. En efecto, cuando una hipótesis no puede aplicarse, se aplica la otra. La hipótesis fonónica emerge como elemento imprescindible debido a que la hipótesis atómica no es universalmente aplicable en todos los fenómenos. En tercer lugar, y por último, el argumento de la cantidad puede cobrar fuerza si recibiera un apoyo teórico adicional de los otros dos argumentos previamente considerados. Sin embargo esto no sucede ya que como demostramos ni el aquí bautizado argumento de la herramienta ni el argumento de la supercomputadora resultan adecuados o eficaces para el caso de los fonones. 


\section{Por lo tanto existen, pero ¿cómo existen?}

La pregunta en torno a la existencia de los fonones cobra relevancia pues como bien afirman Ayer (1936) inicialmente y Quine $(1969,1984)$ posteriormente, el mero hecho de nombrar un objeto no implica su existencia. En este sentido no es posible determinar lo existente simplemente identificando los términos de una teoría. En "Acerca de lo que hay" Quine (1984) apela al análisis lógico propuesto por Russell para analizar las descripciones singulares, con el objetivo de explicar el modo en que podemos utilizar nombres sin referencia objetiva. Según Quine a partir del análisis lógico del lenguaje advertimos que el nombre pasa a ser una variable de un cuantificador existencial al modo de "existe un $x$ tal que (a)". De ser así, la carga de la referencia objetiva se desplaza desde los nombres a las variables ligadas de la lógica. Es decir, la existencia del objeto nombrado no depende del acto de ser nombrado sino del hecho de constituirse como un valor del cuantificador existencial. El criterio de existencia ontológico que Quine establece es el siguiente: "una teoría está obligada a admitir aquellas entidades - y sólo aquellas - a las cuales tienen que referirse las variables ligadas de la teoría para que las afirmaciones hechas en ésta sean verdaderas" (Quine 1984, p.40) Para el autor el peso específico radica en el cuantificador existencial, siendo éste, y no el nombre, el que contiene el importe existencial.

Despejado el nombre como referencia primaria es evidente que la pregunta que resta especificar es qué evidencia cuenta para determinar los objetos que se constituyen como valores de las variables, es decir, qué objetos consideraremos existentes siendo que el mero nombrar ya no es un parámetro para ello. La respuesta de Quine aquí se vuelve pobre: "para esta pregunta no hay una respuesta simple y general. Si la oración abierta debajo del cuantificador es algo así como " $x$ es un conejo" o " $x$ es un unicornio", entonces la evidencia, si la hay, es en gran medida el testimonio de los sentidos." (Quine 1969, p.97). Ahora bien, más allá del modo en que el autor lo formula lo que Quine quiere destacar es que la pregunta por la existencia es un pregunta con sentido que puede hacerse en el marco de una teoría: "Las declaraciones de existencia en esta línea filosófica admiten evidencia, en el sentido de que podemos tener razones, y esencialmente razones científicas, para incluir números o clases o similares en el rango de valores de nuestras variables". (Quine 1969, p.97). Y precisamente esto es lo que intentamos desarrollar en el presente trabajo. Hemos brindado razones científicas y epistémicas que nos permitan afirmar que hay buenas razones para considerar a los fonones como entidades existentes. No sólo demostramos que los fonones son imprescindibles para explicar ciertos fenómenos, y en este sentido en términos quineanos, se los requiere como valor de las variables para hacer verdaderas las oraciones de la teoría, sino que, como demostramos en el apartado anterior, la hipótesis fonónica no tiene ninguna desventaja epistémica con respecto a la hipótesis atómica. 
En este sentido, creemos que estamos brindando un criterio más amplio y más fructífero que el presentado por Hacking. Según el realismo de entidades de Hacking (1983), que se resume en el emblema "si puedes rociarlo, entones son reales" (Hacking 1983, p.83), lo que dirime como condición necesaria y suficiente la potencial existencia de alguna entidad es la capacidad manipulativa. De ser así, entonces las cuasipartículas (familia a la que pertenecen los fonones) existirían por cumplir con tal requisito (Cfr. Gelfert 2010). Sin embargo, nosotros en este trabajo no nos atenemos a este criterio que, tal como afirma Gelfert (2010) puede constituirse como muy estrecho y permisivo a la vez. Atendiendo a la posibilidad de que el éxito manipulativo pueda no ser suficiente, nosotros, al poner en pie de igualdad la hipótesis fonónica con la hipótesis atómica, brindamos criterios explicativos y epistémicos que le confiere a la primera hipótesis una verosimilitud mayor a la que comúnmente se le atribuye.

Ahora bien, precisamente qué es lo que presuponemos cuando afirmamos que algo existe; es decir, qué entendemos por existencia. Para entender el alcance de nuestros propósitos vale la pena acudir nuevamente a Quine: "si atendemos a las variables ligadas en conexión con la ontología no es para saber lo que hay, sino para saber lo que una determinada observación o doctrina, dice que hay" (Quine 1984, p.43). El espíritu de esta expresión nos compele a tratar de entender y elucidar cuál es la ontología propuesta por una determinada teoría. La ontología de una teoría nos dice cuáles son las entidades que pueblan el mundo. Sin embargo somos consciente que esta ontología no puede hacerse desde el punto de vista del Ojo de Dios (Putnam 1981). Es decir, no se realiza desde una perspectiva neutral y absoluta que exponga las entidades que existen independientemente de la mente que los piensa. En términos de Carnap (1950) la pregunta que aquí pretendemos responder no es una pregunta externa sino una pregunta interna: "ser real en el sentido científico es ser un elemento en un marco lingüístico (...) Esta es una pregunta interna. (...). La pregunta externa por la realidad de la cosa en sí misma [... ] no puede ser respondida porque está formulada de modo incorrecto." (Carnap, 1950 p.2). Sin ánimos de considerar en este trabajo si de hecho las preguntas externas tienen o no sentido, lo que Carnap plantea es que las preguntas acerca de la existencia de entidades solo pueden ser formuladas dentro de un determinado marco lingüístico. Este marco lingüístico o conceptual puede ser entendido en términos generales o concretos. Algunos autores (Giere 2006, Fischer 2017) de hecho circunscriben el marco de referencia a partir del cual observamos el mundo a los modelos y a las técnicas experimentales especificas que se utilizan. Otros autores (Lombardi y Pérez Ransanz 2012), en cambio, ponen el foco en ciertos conceptos generales y abstractos como ser la noción de individuo, la noción de espacio, la noción de tiempo o la concepción determinista o indeterminista que se tenga del mundo. Nuevamente no está en los objetivos de este trabajo dirimir tales controversias. Manteniéndonos neutrales en relación ello lo que aquí 
pretendemos demostrar es que el modelo fonónico tiene robustez suficiente como para imponer su propia ontología.

De ser así, entonces creemos haber brindado las razones por las cuales, en función de las teorías científicas vigentes, uno debería aceptar a los fonones como entidades existentes. Sin embargo queda mucho camino por recorrer. La tarea que aún nos queda pendiente es tratar de entender cuál es efectivamente el estatus ontológico de aquellas partículas que llamamos fonones.

Para poder realizar tal cometido y así elucidar el carácter de ser de los fonones se vuelve imprescindible mencionar aquí la distinción entre cuasipartículas y partículas virtuales. La importancia de recordar tales categorías es que nosotros afirmaremos que los fonones, como partículas legítimas, no se ajustan adecuadamente a la forma en que se usualmente se piensan estas descripciones.

Las partículas virtuales aparecen cuando se aplica la teoría de perturbaciones en la teoría cuántica de campos. En teoría de campos el cálculo típico consiste en considerar un grupo de partículas cuyo estado inicial se conoce (estado de entrada), luego dichas partículas son sometidas a un proceso de interacción de duración finita y el objetivo es calcular el estado final luego de la interacción (estado de salida). La forma estándar de hacer esta cuenta consiste en escribir la integral que permite calcular la probabilidad de que dado un estado de entrada obtengamos un estado de salida. Como esta integral no se puede resolver en forma exacta se utiliza la teoría de perturbaciones, que transforma esta integral en una suma infinita de integrales más sencillas. Cada una de estas pequeñas integrales se puede interpretar como la probabilidad de que las partículas de entrada hayan interactuado de una manera particular. A su vez, dicha interacción puede representarse mediante los diagramas de Feynman como el intercambio de una partícula virtual cuántica.

Ahora bien, las razones por las cuales estas partículas son denominadas partículas virtuales son las siguientes. En primer lugar ellas no pueden ser observadas directamente ya que no aparecen en ninguno de los dos estados accesibles al experimentador: no aparecen ni en el estado de entrada ni en el estado de salida. Estas partículas sólo estarían en los procesos intermedios que modelan la interacción En segundo lugar, estas partículas surgen como producto de realizar los cálculos con una técnica matemática determinada que en principio podría no utilizarse. Este último argumento puede sugerir que existe un punto de contacto entre la fonones y las partículas virtuales ya que como expusimos anteriormente el Hamiltoniano fonónico surge a partir de una cambio de coordenadas. Sin embargo esta conclusión es apresurada. Los fonones no son partículas virtuales debido a las siguientes razones. Por un lado, los fonones aparecen en la mecánica cuántica estándar sin necesidad de introducir la teoría cuántica de campos ni la teoría de perturbaciones. Por otro lado, no aparecen como intermediarios de las interacciones de un proceso. Y por último, a diferencia del caso de las partículas virtuales, nada prohíbe que los fonones apa- 
rezcan en los estados de entrada o salida de procesos de scatering (de hecho eso es lo que se hace en el experimento de Shinen - 1963 -). En consecuencia, independientemente a que algunos autores abogan a favor de la existencia de las partículas virtuales (Valente 2011, Jaeger 2019) mientras que otros la niegan (Redhead 1988, Fox 2008), lo que queremos destacar es que por las razones mencionadas se hace necesario considerar el estatus ontológico de los fonones desde una categorización diferente.

Precisamente debido a lo antedicho es que los físicos suelen interpretar a los fonones como cuasipartículas. Las cuasipartículas son entidades definidas en sistemas de muchas partículas interactuando. Generalmente se obtienen a partir de un cambio de variables que permite transformar una descripción en términos de partículas que interactúan por otra descripción que instituye un nuevo tipo de partículas que no interactúan. En la actualidad podemos encontrar más de 30 cuasipartículas que en alguna u otra medida son utilizadas en los cálculos del área materia condensada. Este tipo de entidades suele interpretarse en la literatura actual como meras herramientas de cálculo o ilusiones útiles (Gelfert 2010) o como entidades emergentes (Primas 1983, Ladyman 2015, Franklin y Knox 2018). Siendo que el primer caso ya lo hemos discutido ampliamente ahora trataremos de evaluar los pormenores de la propuesta emergentista.

En la literatura existen dos posturas complementarias. Por un lado, Ladyman en "Are There Individuals in Physics, and If So, What Are They?" en el apartado que trata sobre los individuos emergentes menciona a los fonones como cuasi-partículas, en donde las cuasi-partículas son entidades con un tiempo de vida finito y por lo tanto siempre relativas a una escala de tiempo acotada (Ladyman 2015, p.202). Por otro lado, aunque en continuidad con lo que Ladyman propone, Alexander Franklin y Eleanor Knox (2018) desarrollan un propuesta netamente emergentista para entender el caso de los fonones. En efecto los autores en su artículo "Emergence Without Limits: The Case of Phonons", tratan de explicar la situación ontológica de los fonones dentro de un enfoque emergentista. Considerándose desde esta perspectiva habría un nivel fundamental donde están las entidades basales a partir de las cuales emergen propiedades que no pueden ser explicadas a partir del comportamiento de las entidades básicas. En el nivel de las propiedades emergentes surgen entonces las entidades emergentes. Sin embargo existen algunas dificultades a la hora de aplicar el emergentismo a este caso. En primer lugar, como señalan Franklin y Knox (2018), se trata de un extraño caso de emergencia. Usualmente, al menos en física, el esquema de la emergencia ha sido aplicado mediante la utilización de algún tipo de límite singular o asintótico. Como es posible constatar en una gran cantidad de ejemplos, estos límites nos ayudan a intentar salvar las contradicciones lógicas que hay entre las teorías.

Por otro lado, como en este caso no tenemos límites asintóticos que nos permitan 
aplicar algún truco matemáticos, es necesario explicar las contradicciones lógicas que surgen al aceptar las ontologías del nivel fundamental y el nivel emergente al mismo tiempo. Por ejemplo, en el nivel emergente tenemos núcleos quietos pero en el nivel fundamental hay núcleos cuánticos que de acuerdo al principio de incerteza de Heisenberg no pueden tener la posición y la velocidad bien definida. Entonces aceptar ambos niveles a la vez conduce a una contradicción que el emergentismo, al introducir una jerarquización y una dependencia ontológica entre ellos, no puede convincentemente explicar. Pero además, la contradicción es aún más seria que la que acontece en la aproximación de Born-Oppenheimer (1927) ya que en este caso no podemos aproximar un Hamiltoniano por otro en el límite de alta energía potencial.

Atendiendo a estas dificultades nosotros sospechamos que puede considerarse otra posibilidad que aún no fue suficientemente explorada en la bibliografía específica del caso analizado.

\subsection{El pluralismo ontológico}

Como hemos afirmado las consideraciones presentadas hasta aquí parecen apoyar el hecho de que el estatuto ontológico que se le da a los fonones ha sido devaluado en forma injustificada. Por otro lado, tampoco puede negarse la importancia de los átomos móviles a la hora de explicar los distintos experimentos. En este sentido nos preguntamos si el marco filosófico pluralista no aporta algunas herramientas que nos permita lidiar de un modo más satisfactorio que el emergentismo con esta situación contradictoria que se presenta entre la hipótesis fonónica y la hipótesis atómica usual. No es nuestro objetivo aquí hacer una defensa acérrima de esta perspectiva sino proponerla como una tercera posibilidad frente a la concepción reduccionista aquí descartada y la salida emergentista.

La propuesta pluralista en su versión ontológica si bien reivindicada en los últimos tiempos por autores como Dupré (1993), Cartwright (1994, 1999), Chakravarty (2011) y Olimpia Lombardi y Ana Rosa Pérez Ranzanz (2012) y , suele ser muy resistida al interior de la práctica científica y filosófica. Aun cuando en la práctica científica lo que se observa es una proliferación creciente de modelos configurados a partir de principios teóricos en conflicto (Hendry 1998), o incluso de modelos incompatibles entre sí que se aplican al mismo sistema de estudio, prima, como producto de lo que Scerri (2000) dominó el "imperialismo de la física", una concepción reduccionista, monista y universalista. Tradicionalmente la filosofía de la ciencia se constituyó en miras al devenir de la física, disciplina que se caracterizó por intentar brindar una visión unificada e integral del mundo. Siendo así la tendencia imperante es intentar explicar la multiplicidad de cosas existentes mediante una ontología económica y simple. Sin embargo no sólo a nivel interdisciplinar tal proyecto resulta de difícil concreción debido a la imposibilidad de reducir las diferentes categorías utilizadas, sino 
que también, a nivel intradisciplinar, se presentan obstáculos similares en el interior de la física. En este contexto es donde el pluralismo cobra fuerza ya que, como afirma Chang la mejor razón para ser pluralista es que "no es muy probable que logremos una teoría perfecta que satisfaga todas nuestras necesidades. Si no es probable ello, tiene sentido mantener múltiples sistemas" (Chang 2012, p.20).

Sin embargo, Nancy Carwright no se limita a esta justificación pragmática del pluralismo ni se limita a adoptar un pluralismo en términos puramente epistémicos, sino que haciéndose cargo de lo que la práctica científica actualmente pareciera evidenciar, afirma: "[... ] Como las apariencias sugieren, vivimos en un dappled world (mundo veteado, con lunares), Un mundo rico en cosas, con diferentes naturalezas, comportándose de diferentes modos. "Las leyes que describen este mundo son 'retazos' (are a patchwork), no son piramidales" (Carwright 1999, p.1). En continuidad con esta línea de análisis, aunque desde un enfoque filosófico ligeramente diferente, Olimpia Lombardi y Ana Rosa Pérez Ranzanz adscribiendo a un pluralismo fuertemente ontológico sostienen una versión correspondentista de la verdad: "un realismo de filiación kantiana puede recuperar una forma de correspondencia entendida como adecuación con la ontología constituida desde un cierto esquema conceptual" (Lombardi y Ransanz 2012, p.50). Desde este perspectiva, sería posible entonces afirmar la existencia de entidades y/o propiedades incompatibles siempre que se lo haga desde disimiles esquemas conceptuales. En continuidad con ciertos aspectos fundamentales de la postura de Carnap (1950), lo que las autoras plantean es que la pregunta por lo existente sólo tiene sentido en un sentido interno, ya que en definitiva lo que las teorías científicas describen no es la realidad trascendente sino una ontología que se constituye a partir de un proceso de síntesis entre las esquemas conceptuales y la incognoscible realidad neuménica. Mientras que una postura monista y reduccionista supone que sólo existe una única realidad, la postura pluralista implica no solamente la negación de una realidad absoluta, sino la postulación de múltiples realidades relativas a los diferentes esquemas conceptuales.

\subsubsection{El pluralismo ontológico en la práctica científica: el caso de la química cuántica y el caso de los fonones}

La plausabilidad del pluralismo ontológico se incrementa si uno considera que no es la primera vez que se plantea en la filosofía de la química. De acuerdo con una visión reduccionista tradicional, eminentemente anti-pluralista, las entidades planteadas por la química son apariencias con cierta eficacia explicativa que surgen de una descripción aproximada del único y verdadero mundo descrito por la física cuántica. Sin embargo, la posición reduccionista enfrenta varias dificultades que hacen que tal postura sea de difícil aplicabilidad en el modo en que la práctica científica se desarrolla efectivamente en la actualidad. En efecto, por ejemplo al interior de la química 
cuántica coexisten supuestos incompatibles, provenientes de la química estructural y la mecánica cuántica. Lo relevante del caso es que tales supuestos son irreductibles. Es decir, ciertos conceptos propios de la química estructural clásica, como por ejemplo la noción de estructura molecular, son tan imprescindibles para la práctica química que aún cuando fuera posible una reducción matemática los recursos de la química estructural seguirían siendo absolutamente necesarios. El caso del isomerismo óptico, por ejemplo, plantea que aún logrando calcular el Hamiltoniano exacto de un sistema no se podría obtener a partir de tal información la tan preciada estructura que nos permite diferenciar los isómeros dextrógiros de los levógiros para (para más detalles ver Lombardi 2014). En este sentido desde una perspectiva pluralista la química cuántica se presenta como un cuerpo disciplinar nuevo y autónomo. Un cuerpo autónomo con una ontología propia esencialmente diferente a la ontología instituida por la mecánica cuántica estándar (ver Lombardi y Labarca 2005 y Labarca y Lombardi 2010).

Ahora bien, si analizamos el caso de los fonones advertimos que también puede ser interpretado desde este marco filosófico. En efecto, tanto las descripciones fonónicas como las atómicas, aun siendo incompatibles entre sí, son igualmente válidas en todo contexto . Como ya afirmamos, para el caso que tuviéramos una supercomputadora se podría describir cualquier sistema tanto con un modelo de átomos móviles como con un modelo de fonones. A su vez, aun en el caso actual en el que no disponemos de tal supercomputadora ambas descripciones siguen siendo igualmente válidas en sus respectivos ámbitos de aplicación. En resumen, bajo cualquiera de las dos ópticas analizadas deberíamos aceptar ambas descripciones de la realidad como descripciones válidas.

Entonces desde una perspectiva pluralista, en ciertos contextos, deberíamos admitir una ontología de átomos móviles ya que la misma nos permite explicar una serie de fenómenos. Sin embargo, asumir tal perspectiva en determinados marcos explicativos no debería limitar ni anular otras formas interpretativas. La hipótesis fonónica, en donde los átomos no se mueven, se impone como otra interpretación no excluyente. En este caso son los fonones y no los átomos los que explican, por ejemplo, a partir de su movimiento, el gradiente del calor. Evidentemente ante la pregunta que un realista reduccionista haría respecto a si los átomos efectivamente se mueven o no, el pluralista cambiaría el eje de la pregunta afirmando que la pregunta por las características de lo existente no puede plantearse en términos absolutos ya que, lo que de hecho sucede depende del marco conceptual. Como afirma Cartwright, aunque desde una perspectiva perspectiva contextualista en donde el factor condicionante está dado también, y fundamentalmente, por las situaciones experimentales específicas, "la evidencia indica una conclusión que me resulta agradable - que la naturaleza no es simple ni puede reducirse. Es rica y diversa" (Cartwright, 1994, p.361). 


\section{Conclusiones}

En el presente trabajo hemos intentado analizar y reivindicar el estatus ontológico de los fonones, usualmente desacreditados en la práctica científica como meros elementos de cálculo. Para ello en primer lugar evidenciamos las virtudes epistémicas de tales entidades dando cuenta no sólo de su capacidad explicativa sino también de su capacidad interpretativa respecto a ciertos fenómenos que antes permanecían oscuros.

Luego presentamos una serie de argumentos para explicar por qué no habría razones para no aceptar a los fonones como entidades existentes. Para ello en primer lugar establecimos una analogía entre los fotones y los fonones para que todo aquél que se precie de realista y acepte a los fotones como existentes tenga también que aceptar a los fonones. En segundo lugar refutamos tres argumentos que suelen esgrimirse para explicar la primacía ontológica de los átomos. Explicando las debilidades de los argumento de la herramienta, del argumento de la superconputadora y del argumento de la cantidad, socavamos las bases de defensa de aquellos realistas que pretenden no aceptar el estatus ontológico de los fonones.

Por último, y ya habiendo evidenciado, a partir de las refutaciones de estos argumentos, las dificultades de la postura reduccionista, tratamos de brindar un panorama respecto a las posibles formas de interpretar el estatus ontológico del fonón. Por un lado, explicitamos la perspectiva emergentista ya postulada por ciertos autores para el caso en cuestión, presentado a su vez, ciertas dificultades que tal postura contrae. Por otro lado, introdujimos una nueva propuesta filosófica, el pluralismo ontológico, de modo de poder lidiar con las contradicciones que surgen del caso sin tener que postular una relación jerárquica entre los diferentes niveles de la realidad tal como lo hace el emergentismo.

\section{Referencias}

Amann, A. 1992. Must a molecule have a shape? South African Journal of Chemistry 45: 29-38.

Ashcroft, N. W.; Mermin, N. D. 1976. Solid State Physics. Harcourt College Publishers: Orlando.

Ayer, A. J. 1936. Language, truth and logic. London: Gollancz.

Ballentine, L. E. 1990. Quantum Mechanics. New York: Prentice Hall.

Best, N. W. 2016. Lavoisier's 'Reflections on Phlogiston' II: On the Nature of Heat. Foundations of Chemistry 18: 3-13.

Bishop, R. 2005. Patching physics and chemistry together. Philosophy of Science 72: 716-22. Born, M.; Oppenheimer, J. 1927. On the Quantum Theory of Molecules. Trad. S.M. Blinder, B. Sutcliffe \& W. Geppert [Zur Qantentheorie der Molekeln]. Annalen der Physik 84: 457-84. 
Carnap, R. 1950. Empiricism, Semantics, and Ontology. Revue Internationale de Philosophie 4: 20-40. Reprinted in the Supplement to Meaning and Necessity: A Study in Semantics and Modal Logic. University of Chicago Press: Chicago, 1956.

Cartwright, N. 1994. The metaphysics of the disunified world. Proceedings of the Biennial Meeting of the Philosophy of Science Association 2: 357-64.

Cartwright, N. 1999. The dappled world: A study of the boundaries of science. Cambridge: CUP. Chakravartty, A. 2011. Scientific realism and ontological relativity. The Monist 94: 157-80.

Chang, H. 2012. Is Water $\mathrm{H}_{2} \mathrm{O}$ ? Evidence, Realism and Pluralism. Dordrecht: Springer.

Dirac, P. A. M. 1929. Quantum mechanics of many-electron systems. Proceedings of the Royal Society of London. Series A 123(792): 714-33.

Dupré, J. 1993. The Disorder of Things: Metaphysical Foundations of the Disunity of Science. Cambridge MA: HUP.

Franklin, A.; Eleanor Knox. 2018. Emergence Without Limits: The Case of Phonons. Studies in History and philosophy of Modern Physics 64: 68-78.

Fischer, G. 2017. Content, design, and representation in chemistry. Foundations of Chemistry 19: 17-28.

Fox, T. 2008. Haunted by the Spectre of Virtual Particles: A Philosophical Reconsideration. Journal for General Philosophy of Science 39: 35-51.

Gelfert, A. 2010. Manipulative success and the unreal. International Studies in the Philosophy of Science 17: 245-63.

Giere, R. 2006. Perspectival Pluralism. In: S. Kellert; H. Longino; K. Waters (eds.) Scientific Pluralism, pp.26-41. London: University of Minnesota Press.

Hacking, I. 1983. Representing and Intervening: Introductory Topics in the Philosophy of Natural Science. Cambridge: Cambridge University Press.

Hendry, R. F. 1998. Models and approximation in Quantum Chemistry. Poznan Studies in the Philosophy of Science and the Humanities 63: 123-42.

Hendry, R. F. 2010.Ontological Reduction and Molecular Structure. Studies in History and Philosophy of Science Part B: Studies in History and Philosophy of Modern Physics 41(2): 183-91.

Hettema, H. 2012. Reducing Chemistry to Physics. Limits, Models, Consequences. Groningen: Rijksuniversiteit Groningen.

Kittel, C. 1998. Introducción a la física del estado sólido. Reverté: Barcelona.

Jaeger, G. 2019. Are Virtual Particles Less Real? Entropy 21(2): 1-17.

Kong, Y.; Dolgov, O. V.; Jepsen, O.; Andersen, O. K. 2001. Electron-phonon interaction in the normal and superconducting states of MgB2. Physical Review B 64(2): 020501-4.

Labarca, M.; Lombardi, O. 2010. Why orbitals do not exist? Foundations of Chemistry 12: 149-57.

Ladyman, J. 2015. Are there Individuals in Physics, and If So, What Are They? In: A. Guay; T. Pradeu (eds.) Individuals across the Sciences, pp.193-206. Oxford University Press.

Lombardi, O. 2014. Linking chemistry with physics: arguments and counterarguments. Foundations of Chemistry 16: 181-92.

Lombardi, O.; Labarca, M. 2005. The ontological autonomy of the chemical world. Foundations of Chemistry 7: 125-48.

Lombardi, O.; Pérez Ransanz, A. R. 2012. Los Múltiples Mundos de la Ciencia. Un Realismo Pluralista y su Aplicación a la Filosofía de la Física. México: UNAM-Siglo XXI. 
Putnam, H. 1981. Reason, Truth and History. Cambridge: Cambridge University Press.

Quine, W. 1969. Ontological Relativity and Other Essays. New York: Columbia University Press.

Quine, W. 1984. Acerca de lo que hay. In: Desde un punto de vista lógico, pp.25-47.Trand. M. Sacristán [From a Logical Point of View] Barcelona:Ediciones Ariel.

Primas, H. 1983. Chemistry, Quantum Mechanics and Reductionism. Berlin: Springer-Verlag

Redhead, M. 1988. A philosopher looks at quantum field theory. In: H. R. Brown; R. Harré (eds.) Philosophical foundations of quantum field theory, pp.9-24. Oxford: Clarendon Press.

Sakurai, J. J. 1994. Modern Quantum Mechanics. Addison-Wesley: New York.

Scerri, E. 2000. The failure of reduction and how to resist disunity of the sciences in the context of chemical education. Science \& Education 9: 405-25.

Scerri, E. R. 2011. Editorial 37. Foundations of Chemistry 13: 1-7.

Scerri, E. R. 2013. Philosophy of chemistry: where has it been and where is it going. In: J.-P. Llored (ed.) The Philosophy of Chemistry: Practices, Methodologies, and Concepts, pp.20825. Newcastle: Cambridge Scholars Publishing.

Shinen, N. S. 1963. Nonlinear acoustic interaction in $\mathrm{MgO}$ at $9 \mathrm{Gc} / \mathrm{sec}$. Physical Review Letters 11: 3-6.

Sutcliffe, B. T.; Woolley, R. G. 2011. A comment on Editorial 37. Foundations of Chemistry 13: 93-5.

Sutcliffe, B. T.; Wolley, R. G. 2012. Atoms and molecules in classical chemistry and quantum mechanics. In: R. F. Hendry; P. Needham; A. Woody (eds.) Handbook of Philosophy of Science. Philosophy of Chemistry. Volume. 6, pp.387-426. Elsevier: Oxford.

Valente, M. B. 2011. Are Virtual Quanta Nothing but Formal Tools? International Studies in the Philosophy of Science 25: 39-53.

Woolley, R. G. 1978. Must a molecule have a shape? Journal of the American Chemical Society 100: 1073-8.

Woolley, R. G. 1982. Natural optical activity and the molecular hypothesis. Structure and Bonding 52: 1-35. 